\title{
NATURAL HISTORY NOTE \\ Primera colonia de nóctulos medianos (Nyctalus noctula Schreber, 1774) en Castilla y León (Norte de España)
}

\author{
Manuel Fabio Flechoso del Cueto ${ }^{1,}{ }^{*}$, Daniel Fernández Alonso, Juan Tomás Alcalde Díaz de Cerio
}

\author{
${ }^{1} \mathrm{C} /$ Palencia n으 6, 1ㅇF 49022 \\ Zamora.
}

*Corresponding author:

fabioflechoso@hotmail.com

DOI: https://doi.org/10.14709/ BarbJ.11.1.2018.02

Palabras clave: Castilla y León, Nóctulo mediano, conservación Nyctalus noctula, Soria.

Keywords: Castilla y León, Common noctule, conservation, Nyctalus noctula, Soria.

received: February, 12th 2018 accepted: April, 3rd 2018

\begin{abstract}
RESUMEN
Hasta ahora sólo se tiene constancia de cinco agrupaciones de nóctulos medianos (Nyctalus noctula) en la península Ibérica, muy distantes entre sí, situadas en Navarra (dos), Zaragoza, Lleida y Madrid. En septiembre de 2016 se capturó un macho en el Parque de la Arboleda de Almazán (Soria) a orillas del río Duero. En septiembre también, se identificaron nueve refugios utilizados por esta especie mediante la detección de sonidos sociales emitidos por los machos, ocho en álamos (Populus alba) y uno en un chopo negro (Populus nigra), todos ellos en nidos abandonados de pícidos (Fam. Picidae). En junio de 2017 se observó la salida de 23 ejemplares de otro nido de pícido abandonado, situado en un álamo. Mediante redes y arpas, se capturaron tres únicos individuos, también machos. Los datos obtenidos permiten deducir la presencia de un grupo de machos sedentarios en este parque, probablemente acompañados por hembras en otoño. Se trata de la primera colonia de esta especie identificada en Castilla y León y la de mayor altitud (960 msnm) en la península Ibérica. Su situación geográfica puede ser estratégica, al encontrarse entre las colonias del Norte y Centro de la península, por lo que podrían albergar ejemplares de ambas zonas durante sus movimientos migratorios. Por todo ello se considera un bosque relevante para la especie, cuya conservación es prioritaria.
\end{abstract}

\begin{abstract}
Up to now, only five colonies of common noctule (Nyctalus noctula) have been recorded in the Iberian peninsula. They are located very distant from each other, in Navarra (two), Zaragoza, Lleida and Madrid. In September 2016 a male was trapped in the Almazán's La Arboleda park (Soria), on the banks of the Duero river. In September too, nine roosts used by this species were identified by detecting their male social calls, eight in white poplars (Populus alba) and one in a black poplar (Populus nigra). All of them were observed in abandoned nests of Picidae. In June 2017, 23 indviduals were observed leaving another Picidae abandoned nest in a white poplar. Using mist-nets and harp-traps, three individuals were captured, all males. The obtained data allow us to deduce the presence of a group of sedentary males in this park, which are probably accompanied by females in the fall. This is the first colony of common noctule identified in Castilla y León and the highest one (960 masl) in the Iberian peninsula. Its geographical location can be strategic, between the colonies of the North and Center of the peninsula, reason why it could include noctules from both areas during their migratory movements. Therefore, it is considered a relevant forest for this species, whose conservation is a priority.
\end{abstract}

El nóctulo mediano (Nyctalus noctula) es un murciélago de tamaño grande, arborícola y migrante (Sluiter \& Van Heerdt 1966, Rachwald 1992, Strelkov 2000). Se cataloga como especie vulnerable en el Catálogo Español de Especies Amenazadas (RD 139/2011). En la península Ibérica solo se conocen cinco pequeñas agrupaciones de esta especie, distribuidas por el centro y norte: Aranjuez en Madrid (Ruedi et al. 1998), Pamplona y Sangüesa en Navarra (Alcalde 1999), Lleida (Camprodon \& Guixé 2013) y Nuévalos en Zaragoza (Lorente et al. 2014). Únicamente la primera es reproductora; las demás están formadas exclusivamente por machos sedentarios en verano, que se ven acompañados de hembras a partir de finales de agosto hasta bien entrado el otoño. Durante el periodo de apareamiento los machos en celo son territoriales y emiten potentes chillidos desde sus refugios, lo que facilita su localización (Kronwitter 1988, Alcalde 2008). Todavía se desconoce el origen de estas hembras, que pasan el invierno en la península (Alcalde 2008) aunque se sospecha que provienen de colonias reproductoras asentadas en el continente Europeo, como se ha comprobado con hembras de nóctulo pequeño, Nyctalus leisleri (Ohlendorf et al. 2000, Alcalde et al. 2013a). 


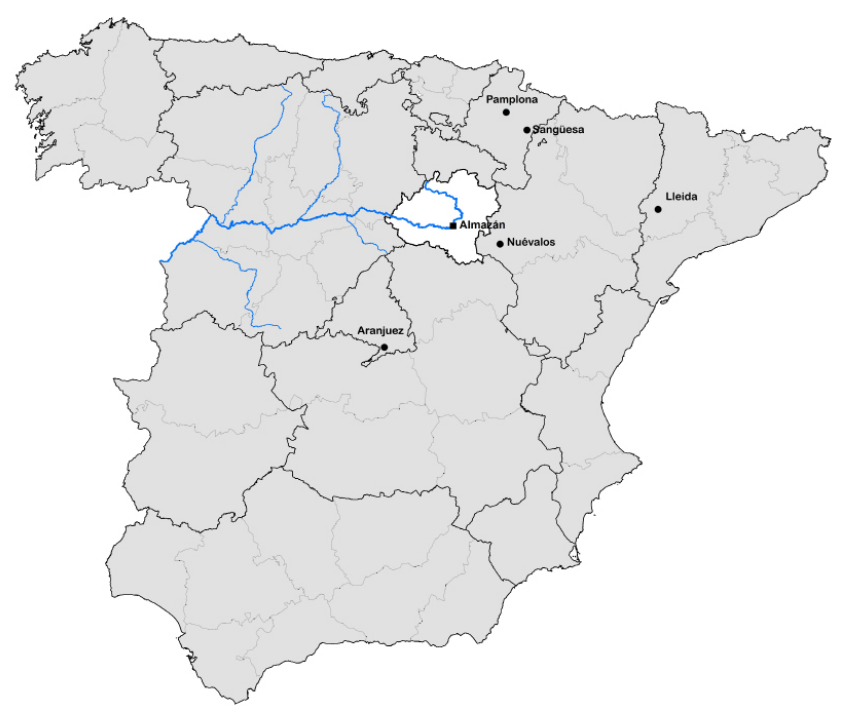

Fig.1 - Localización de la colonia de Almazán junto al Río Duero (en azul) y las demás agrupaciones conocidas en España.

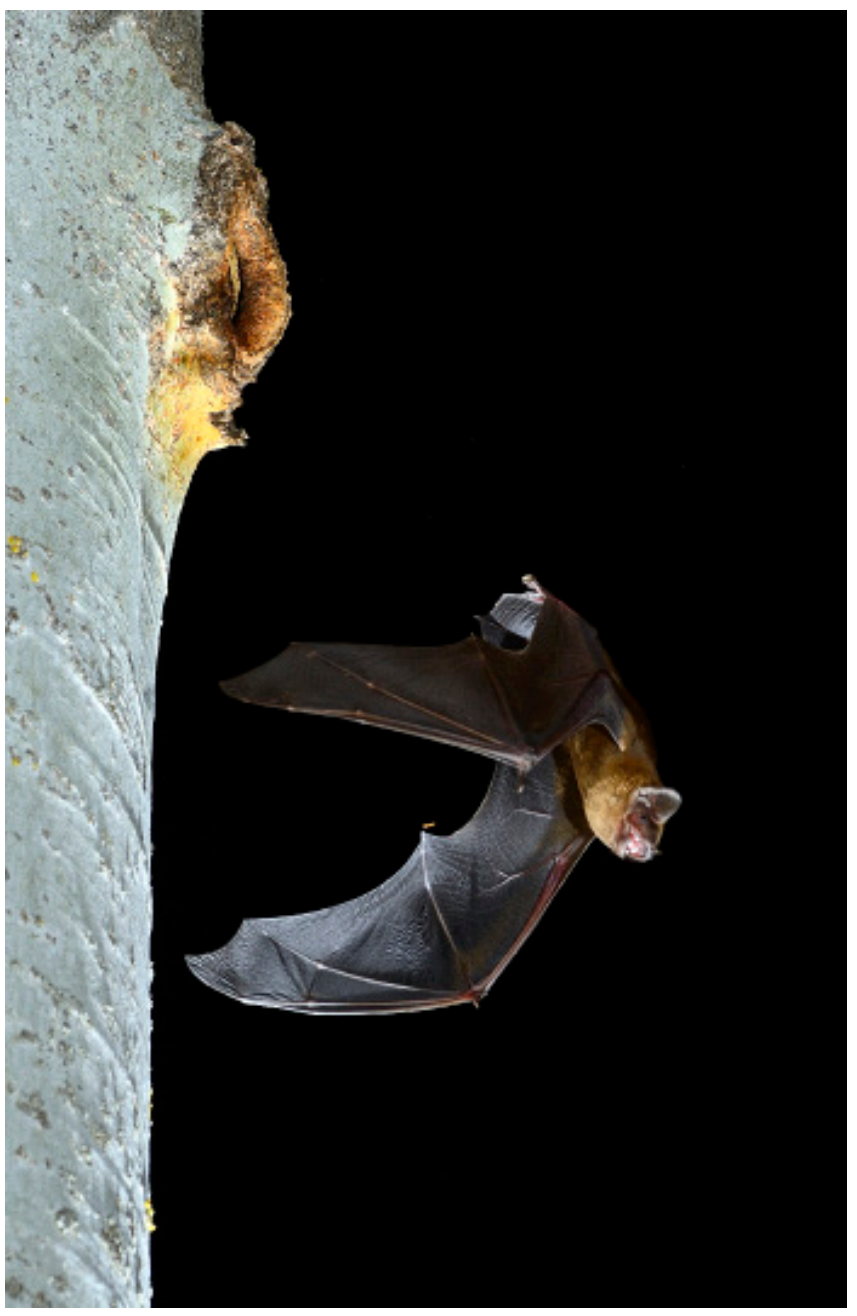

Fig. 2 - Nóctulo mediano ( $N$. noctula) fotografiado a la salida de su refugio en un viejo nido de pícido ubicado en un álamo blanco (La Arboleda, Almazán). Autor J. M. Castrillo.

Todas las agrupaciones halladas hasta ahora se encuentran en parques con arbolado viejo y de grandes dimensiones, situados junto a tramos lentos de ríos. La altitud de estas zonas oscila entre 170 y $750 \mathrm{msnm}$. El parque de La Arboleda se encuentra en Almazán, a orillas del río Duero, a 960 msnm (UTM X: 538603; Y: 4592960. Datum ETRS89, Fig. 1). Comprende una superficie aproximada de

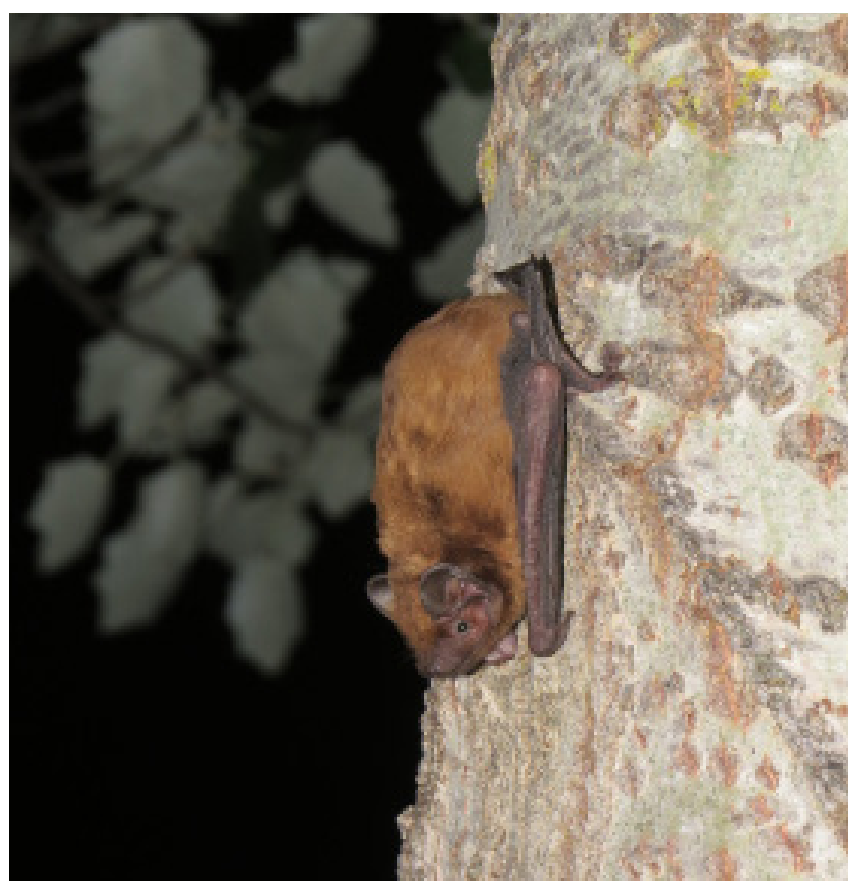

Fig. 3 - Nóctulo mediano ( $N$. noctula) fotografiado después de ser capturado y puesto en libertad (La Arboleda, Almazán). Autor M. Fabio Flechoso.

$85.000 \mathrm{~m}^{2}$ y las especies arbóreas más abundantes son álamos (Populus alba) y chopos negros (Populus nigra), aunque también hay alisos (Alnus glutinosa), castaños de indias (Aesculus hippocastanum), sauces (Salix sp.) y algunas coníferas autóctonas y exóticas. El parque contiene numerosos árboles maduros de gran porte con abundantes nidos de pícidos (Fam. Picidae).

Entre agosto y septiembre de 2016 se prospectó el Parque de la Arboleda en busca de refugios de murciélagos en los árboles mediante la escucha de sonidos sociales producidos por nóctulos (Alcalde 2008, Camprodon et al. 2014), en recorridos realizados a pie, entre las 19 y las 22:30 horas, ayudados de un detector de ultrasonidos en heterodino (Pettersson D230) a frecuencias de entre 18 y 25 $\mathrm{kHz}$. Por la noche se realizaron grabaciones de ultrasonidos en tiempo expandido (micrófono Pettersson M 500-384 conectado a teléfono móvil). Los ultrasonidos se analizaron con el programa informático Bat Explorer (Elekon). También se tomaron fotografías de ejemplares en vuelo utilizando una cámara réflex conectada a flashes y fotocélulas (Fig. 2) para identificar individuos sin capturarlos. El censo de un grupo de nóctulos se realizó utilizando una cámara de vídeo con focos de luz infrarroja y detector de ultrasonidos. Al anochecer se utilizaron redes finas y trampas de arpa pequeñas, colocadas a la salida de los refugios para capturar ejemplares. Los individuos capturados se midieron, pesaron y anillaron con anillas específicas para nóctulos medianos (tamaño 3x) del Ministerio de Agricultura y Pesca, Alimentación y Medio Ambiente, para cuyo uso con esta especie se ha contado con la correspondiente autorización de la Asociación Española para la Conservación y el Estudio de los Murciélagos.

El 22 de agosto de 2016 se escucharon sonidos sociales típicos de murciélagos en un nido abandonado de pícido, situado en un álamo, a dos metros de altura. El 24 del 


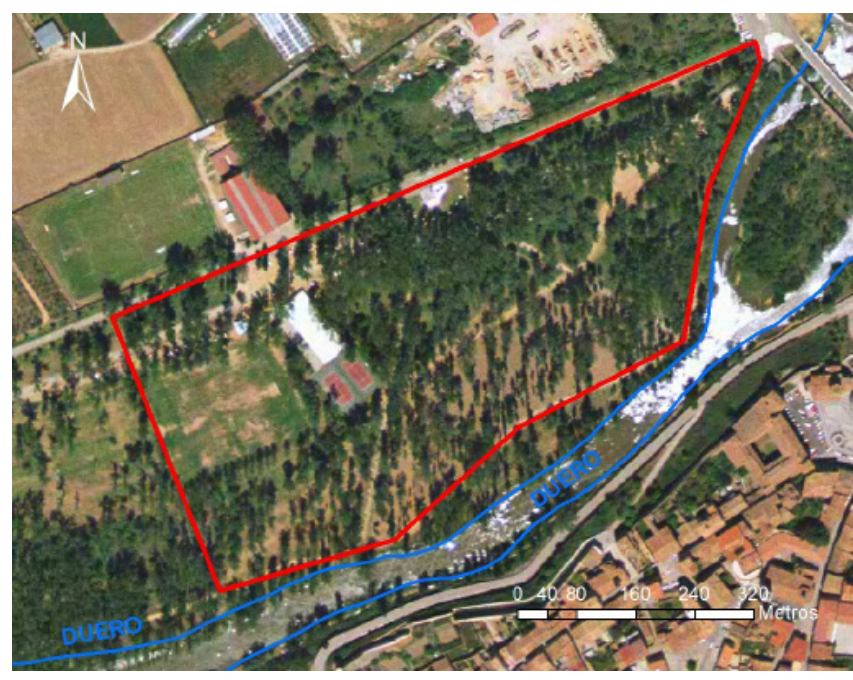

Fig. 4 - Plano del contorno del parque de La Arboleda de Almazán que ha sido estudiado y donde se han encontrado los refugios de N. noctula.

mismo mes se grabó en vídeo la salida de un murciélago, observándose que era un nóctulo de tamaño grande. El seis de septiembre se capturó el ejemplar con una red y se comprobó que era un macho de $N$. noctula (Fig. 3). El individuo capturado emitió potentes sonidos sociales durante su examen y otros cinco ó seis nóctulos que salieron de un refugio cercano, volaron alrededor del ejemplar mostrando aparente curiosidad. El nueve de septiembre se revisó el parque al atardecer y se escucharon sonidos sociales similares en nueve refugios dentro del contorno del propio parque (Fig. 4). Todos los refugios encontrados eran nidos abandonados de pícidos y se hallaban en álamos blancos, salvo uno, ubicado en un chopo negro. Solamente uno de los refugios se localizaba en un árbol muerto. La mayoría de los huecos presentaba la entrada desgastada y con coloración pardo-amarillenta. El 11 de septiembre se tomaron varias fotografías de los ejemplares que salían de dos de estos refugios, comprobándose que se trataba de nóctulos medianos. El refugio inicial mencionado anteriormente contenía un individuo, mientras que otro, situado a cuatro metros del anterior albergaba seis nóctulos.

El año siguiente, el 13 de junio de 2017 se llevó a cabo una nueva revisión del parque, localizándose otro nido abandonado de pícido en un álamo, a cuatro metros de altura, en el que se escucharon numerosos sonidos sociales. Al anochecer se grabó en vídeo la salida de 23 ejemplares. El 19 del mismo mes se capturaron tres individuos mediante trampa de arpa colocada junto a la boca del refugio. Todos ellos eran nóctulos medianos machos, con los testículos escrotales (Tabla 1).
Las observaciones y capturas realizadas en Almazán ponen de manifiesto la presencia de una población residente de nóctulos medianos en Soria, que es además la única encontrada hasta ahora en Castilla y León. Por los datos recogidos, la colonia hallada muestra una fenología similar a las presentes en Navarra y Aragón (Alcalde 2008, Lorente et al. 2014): en junio, la población está formada únicamente por machos, algunos de los cuales pueden reunirse en un mismo hueco; pero en septiembre, estos adoptan un comportamiento territorial y se reparten por diferentes refugios, lo que permite suponer el comienzo de la época de apareamientos con hembras migrantes. La presencia de un grupo de seis ejemplares saliendo de un mismo refugio contiguo al del primer macho capturado en septiembre de 2016, cuando los machos ya se han dispersado, hace suponer la presencia de al menos un harén. Sería conveniente realizar nuevos muestreos en otoño para aclarar esta hipótesis.

La colonia encontrada contiene un mínimo de 23 machos en junio, que probablemente se verá reforzada por nuevos machos y hembras en septiembre. Aunque el grupo probablemente no es muy numeroso, parece ser similar a los encontrados en otros territorios como Sangüesa (Alcalde 1999), Lleida (Camprodon \& Guixé 2013) o Nuévalos (Lorente et al. 2014) donde las colonias también cuentan con pocos ejemplares.

La observación de machos en dos años consecutivos, así como el aspecto de la boca de algunos refugios, que se encuentran desgastados y con un tono pardo-amarillento, característico del paso de los murciélagos (Gebhard \& Bogdanowiccz 2004), permite suponer que la colonia utiliza regularmente este parque y por tanto su presencia no es ocasional.

El hábitat en el que se encuentra la colonia es muy similar al descrito para otras agrupaciones de esta especie, como las de Aranjuez (Ruedi et al. 1998), Pamplona y Sangüesa (Alcalde 1999), o Lleida (Camprodon \& Guixé 2013). Se trata de un parque urbano, con árboles frondosos de gran tamaño con abundantes oquedades, cercano al curso bajo de un río. Ello indica la preferencia de los nóctulos medianos por este tipo de hábitat y convendría tratar de localizar nuevas agrupaciones de este raro murciélago en zonas similares.

La colonia de Almazán se sitúa en un lugar geográficamente estratégico, relativamente cerca del grupo de Nuévalos (70 km), y en posición intermedia entre las agrupaciones situadas más al norte (Navarra y Lleida) y la de Aranjuez, todas ellas a más de $150 \mathrm{~km}$ de Almazán (Fig. 1). Por ello, su mantenimiento puede ser muy relevante para permitir el contacto de estas poblaciones que se encuentran

Tabla 1 - Datos biométricos de los ejemplares de N. noctula capturados en el parque de La Arboleda (Almazán).

\begin{tabular}{lcccc}
\hline Fecha captura & Anilla & Sexo & Peso $(\mathrm{g})$ & Antebrazo $(\mathrm{mm})$ \\
\hline $\mathbf{0 6 / 0 9 / 2 0 1 6}$ & - & Macho & - & 54,7 \\
$\mathbf{1 9 / 0 6 / 2 0 1 7}$ & $3 \times 14378$ & Macho & 24,6 & 52,6 \\
$\mathbf{1 9 / 0 6 / 2 0 1 7}$ & $3 \times 14380$ & Macho & 30,7 & 54,2 \\
$\mathbf{1 9 / 0 6 / 2 0 1 7}$ & $3 \times 14381$ & Macho & 27,8 & 54,9 \\
\hline
\end{tabular}


muy distantes entre sí. Cabe destacar también la elevada altitud a la que se encuentra (960 msnm), muy superior a las registradas en las demás colonias de la península y de Europa, donde suele habitar por debajo de los $550 \mathrm{msnm}$ (Dietz \& Kiefer 2017).

En todo caso, la presencia de grupos estables de nóctulos medianos en esos parques, que son intensamente manejados por servicios de jardines, exige una atención particular para asegurar la conservación de este escaso y amenazado murciélago en España, así como sus refugios. Para ello es preciso identificar y conservar los árboles utilizados por la agrupación. Dado que se trata de un parque público, frecuentado por transeúntes, debería realizarse un esfuerzo para compatibilizar el mantenimiento de esta agrupación con el uso recreativo y seguro por las personas que lo visitan. Al menos uno de los árboles utilizados está muerto, por lo que es previsible su tala en breve. Para tratar de mantener los refugios naturales al tiempo que disminuir riesgos para viandantes, se recomienda la poda parcial de los árboles más frágiles, rebajando su altura o retirando las ramas más inclinadas. Ello les proporcionará mayor estabilidad y alargará su vida. Si algún árbol con huecos requiriese ser cortado por entrañar peligro, sería conveniente realizar revisiones previas para asegurarse de que no hay nóctulos en su interior e instalar cajas-refugio (como por ejemplo el modelo $2 \mathrm{FN}$ de Schwegler) que compensaran al menos en parte, la disminución de refugios naturales. Se ha comprobado la utilización de este modelo por esta especie en Pamplona (Alcalde et al. 2013b).

También se aconseja la plantación de nuevos árboles, preferiblemente álamos y chopos, que sustituyan a los talados y en el futuro puedan constituir nuevos refugios para los nóctulos medianos.

\section{AGRADECIMIENTOS}

Especialmente a los Agentes Medioambientales de la Junta de Castilla y León Christian Pascual, Andrés García y Roberto Soria, así como a Mariano García y Fernando García. A todos ellos por sus observaciones, ayuda y colaboración durante los muestreos y captura de ejemplares. Al Servicio Territorial de Medio Ambiente de Soria por la obtención de la autorización y permiso de captura de los ejemplares. A Víctor Salvador por la cesión del detector de ultrasonidos Pettersson D230. A José Manuel Castrillo por su colaboración en la realización de las fotografías realizadas de los ejemplares saliendo de sus refugios, así como por la cesión de las mismas. Finalmente a Lucía Flechoso, cuya presencia produjo el hallazgo casual de los primeros ejemplares.

\section{REFERENCIAS}

ALCALDE, J. T. (1999). New ecological data on the noctule bat (Nyctalus noctula Schreber, 1774) (Chiroptera, Vespertilionidae) in two towns of Spain. Mammalia, 63: 273-280. https://doi.org/10.1515/mamm.1999.63.3.273

ALCALDE, J. T. (2008). El nóctulo mediano en Pamplona. Biodiversidad Urbana de Pamplona. Ayuntamiento de Pamplona - Iruñeko Udala. 50 pp.
ALCALDE J. T., IBÁÑEZ C., ANTÓN I. \& NYSSEN P. (2013a). First case of migration of a Leisler's bat (Nyctalus leisleri) between Spain and Belgium. Le Rhinolophe, 19: 87-88.

ALCALDE J. T., CAMPION D., FABO J., MARÍN F., ARTÁZCOZ A., MARTÍNEZ I., \& ANTÓN I. (2013b). Ocupación de cajas-refugio por murciélagos en Navarra. Journal of Bat Research \& Conservation, 6 (1): 34-43. https://doi. org/10.14709/BarbJ.6.1.2013.05

CAMPRODON, J. \& GUIXÉ, D. (2013). Estado poblacional, selección de refugios y ecología espacial de las poblaciones de nóctulo grande (Nyctalus lasiopterus) y nóctulo mediano (Nyctalus noctula) en Cataluña. Journal of Bat Research \& Conservation, 6 (1): 51 - 59. https:// doi.org/10.14709/BarbJ.6.1.2013.07

DIETZ C., \& KIEFER, A. (2017). Murciélagos de Europa. Editorial Omega, Barcelona, España, 400 pp.

GEBHARD, J. \& BOGDANOWICZ, W. (2004). Nyctalus noctula (Schreber, 1774) Großer Abendsegler. In: Handbuch der Säugetiere Europas, Band 4/II. Aula Verlag, Wiebelsheim, Alemania, pp. 605-694.

KRONWITTER, F. (1988). Population structure, habitat use and activity patterns of the noctule bat, Nyctalus noctula Schreb., 1774 (Chiroptera, Vespertilionidae) revealed by radio-tracking. Myotis, 26: 23-85.

LORENTE, L., LLANA, C., \& SÁNCHEZ, J. M. (2014). Primera cita de nóctulo mediano (Nyctalus noctula) en Aragón (NE de España). Journal of Bat Research \& Conservation, 7(1): 19-21. https://doi.org/10.14709/BarbJ.7.1.2014.04

OHLENDORF V. B., HECHT B., STRASSBURG D. \& AGIRREMENDI P. T. (2000). Fernfund eines Kleinabendseglers (Nyctalus leisleri) in Spanien. Nyctalus, 7 (3): 239-242.

RACHWALD, A. (1992). Habitat preference and activity of the noctule bat Nyctalus noctula in the Bialowieza Primeval Forest . Acta Theriologica, 37: 413-422. https:// doi.org/10.4098/AT.arch.92-42

RUEDI, M., TUPINIER, Y., \& DE PAZ, O. (1998). First breeding record for the noctule bat (Nyctalus noctula) in the Iberian Peninsula. Mammalia, 62: 301-304.

SLUITER, J. W. \& VAN HEERDT, P. F. (1966). Seasonal habits of the noctule bat (Nyctalus noctula). Archives Néerlandaises de Zoologie, 16(1): 423-439. https://doi. org/10.1163/036551666X00011

STRELKOV, P. P. (2000). Seasonal distribution of migratory bat species (Chiroptera, Vespertilionidae) in eastern Europe and adjacent territories. Myotis, 37: 7-25. 